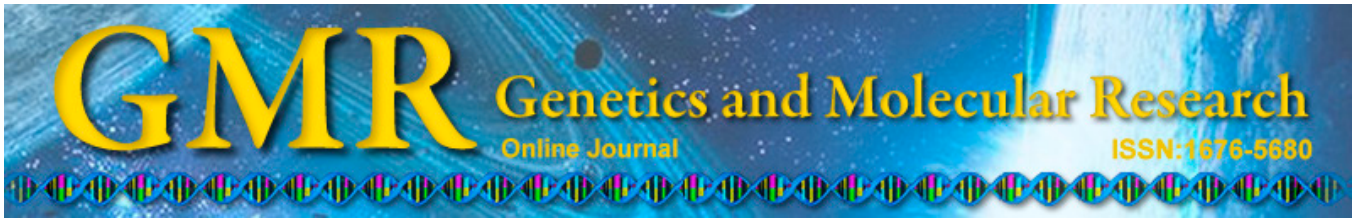

\title{
Expression analysis of Gli1 and Gli2 in different tissues and muscle-derived cells of Qinchuan cattle
}

\author{
Y. Liu ${ }^{1}$, H. Wang ${ }^{1,2}$, G. Cheng ${ }^{1,2}$, B. Jiang ${ }^{1}$ and L. Zan ${ }^{1,2}$ \\ ${ }^{1}$ College of Animal Science and Technology, Northwest A\&F University, \\ Yangling, Shaanxi, China \\ ${ }^{2}$ National Beef Cattle Improvement Center, Northwest A\&F University, \\ Yangling, Shaanxi, China \\ Corresponding author: L. Zan \\ E-mail: zanlinsen@163.com
}

Genet. Mol. Res. 13 (4): 8767-8775 (2014)

Received October 8, 2013

Accepted April 24, 2014

Published October 27, 2014

DOI http://dx.doi.org/10.4238/2014.October.27.18

ABSTRACT. The Hedgehog (Hh) signaling pathway regulates the
differentiation of many kinds of cells and plays a critical role in many
embryonic and postnatal developmental processes. Gli1 and Gli2 are
two transcription factors of the Hh signaling pathway. In this study,
we used quantitative real-time polymerase chain reaction to detect the
relative expression of Gli1 and Gli2 in 13 tissues from three two-year-
old purebred Qinchuan cattle, as well as in different cell populations
derived from muscle and different stages of myogenic differentiation
of myoblasts. The expression levels of Gli1 and Gli2 in muscle were
the lowest of the 13 tissues ( $\mathrm{P}<0.05$ ), and they declined predominantly
from preplate (pp) 1 to pp6 cells. However, the expression of Gli2
was elevated during myogenic differentiation until the 6th day. We
speculated that Hh signaling was negatively activated in myocytes and
quiescent myoblasts. The increased expression of Gli1 and Gli2 in the
early days of myogenic differentiation suggested that Hh signaling
would be activated when the quiescent bovine myoblast was stimulated 
to initiate myogenic differentiation.

Key words: Hedgehog signaling pathway; Myogenic differentiation; Myoblast; Quantitative real-time polymerase chain reaction

\section{INTRODUCTION}

The Hedgehog $(\mathrm{Hh})$ signaling pathway has been thought to be critical to many developmental and growth processes (Varjosalo and Taipale, 2008; Schaefer et al., 2013). Aberrant Hh signaling can cause several kinds of cancer and congenital defects (Fontaine et al., 2008). The ligands of the Hh signaling pathway are secreted proteins, which usually act as morphogens that signal target cells according to a concentration gradient to influence their fate in the development of many parts of the body (Ingham and McMahon, 2001; Hooper and Scott, 2005). Sonic hedgehog (Shh), Indian hedgehog (Ihh), and Desert hedgehog (Dhh) are three Hh proteins that have been identified in mammals.

When the ligand Hh is absent, Patched1 (Ptch1) represses Smoothened (Smo) activity, and the transcription factor Gli enters the nucleus as the repressor form (Gli-R) and blocks the transcription of target genes (Milla et al., 2012). The binding of Hh to Ptch1 can remove the inhibition of Smo. Activated Smo enables the release of Gli from a repressor complex. The activated form of Gli (Gli-A) binds to the regulation region and causes the expression of the target gene. The relative ratio of Gli-A to Gli-R is thought to be crucial for transducing the extracellular Hh signal and for determining concentration-dependent cell fates (Persson et al., 2002; Bai et al., 2004).

Many studies support the hypothesis that activating the Hh signaling pathway by an agonist or overexpressing its positive regulator promoted the differentiation of mesenchymal stem cells (MSCs) into chondrocytes (Warzecha et al., 2006; Li et al., 2012; Steinert et al., 2012) and osteoblasts (Dwyer et al., 2007; Ho et al., 2007; James et al., 2010; Cai et al., 2012a,b; Oliveira et al., 2012), but it inhibited adipocyte differentiation (Fontaine et al., 2008; Cai and Deng, 2010; James et al., 2010). Additionally, the repression of the Hh signaling pathway induced by cyclopamine or a small interfering RNA (siRNA) of its positive component inhibited chondrogenic differentiation (Wu et al., 2013). The Hh signaling pathway promotes myogenesis in zebrafish (Flynt et al., 2007; Lobbardi et al., 2011), Xenopus (Martin et al., 2007), mouse (Straface et al., 2009; Hu et al., 2012; Voronova et al., 2013), and chick (Elia et al., 2007).

Although the Hh signaling pathway has been studied in multiple tissues and cells, the relative expression levels in different tissues in many species are still unclear. Cattle are the main type of livestock in the world, and their muscles are an important food for humans. This study aimed to determine the relative expression levels of the two transcription factors Gli1 and Gli2 and their expression patterns in different populations of cells derived from muscle and in different stages of myoblast differentiation.

\section{MATERIAL AND METHODS}

\section{Sample collection}

Samples of 13 tissues including heart, liver, spleen, lung, kidney, rumen, reticulum, omasum, abomasum, small intestine, large intestine, muscle, and abdominal fat were obtained 
from three two-year-old purebred Qinchuan cattle (Experimental Farm of National Beef Cattle Improvement Center, Yangling, Shaanxi, China) after slaughter. All samples for RNA extraction were promptly frozen in liquid nitrogen and stored at $-80^{\circ} \mathrm{C}$ after being washed three times in phosphate-buffered saline (PBS).

\section{Preparation of different populations of muscle-derived cell cultures}

The mass of fresh longissimus muscle was taken to laboratory from the butchery within $2 \mathrm{~h}$ in sterile PBS containing 2\% penicillin-streptomycin (Gibco, United States). Muscles were minced into a coarse slurry with a fine scissors, enzymatically dissociated at $37^{\circ} \mathrm{C}$ in $0.2 \%$ collagenase type I (Sigma-Aldrich, United States) for $3 \mathrm{~h}$, and centrifuged at 1500 $\mathrm{rpm}$ for $5 \mathrm{~min}$. The supernatant was removed, and the cell pellet was resuspended and washed with Dulbecco's modified Eagle medium (DMEM) (Gibco) containing 10\% fetal bovine serum (FBS) (PAA, Germany) and 2\% penicillin-streptomycin (Gibco). Resuspended cells were filtered with a $40-\mu \mathrm{m}$ cell mesh. In order to isolate the different populations of muscle-derived cells, we adopted the method from Qu-Petersen et al. (2002). The filtered muscle cells were centrifuged, resuspended in proliferation medium [PM: DMEM $+20 \% \mathrm{FBS}+10 \%$ horse serum (HS) (Gibco)] containing 1\% penicillin-streptomycin, and plated on collagen-coated dishes (collagen type I; Sigma-Aldrich) for $2 \mathrm{~h}$ (preplate 1, pp1). The nonadherent cells were then transferred to other dishes (pp2). After $24 \mathrm{~h}$, the suspended cells in pp2 were transferred to new dishes (pp3) to incubate for another $24 \mathrm{~h}$. Then, the suspension was centrifuged, resuspended in fresh medium, and plated on new dishes (pp4). Pp5 and pp6 were obtained in the same way. All dishes were replaced with fresh medium every $48 \mathrm{~h}$, and cells were cryopreserved or collected for RNA extraction when the cells reached $70 \%$ confluence.

\section{Differentiation of myoblasts}

The pp6 cells were digested with $0.05 \%$ trypsin-ethylenediaminetetraacetic acid (Gibco), and centrifuged at $1000 \mathrm{rpm}$ for $5 \mathrm{~min}$. The cells were resuspended in PM and then seeded into Petri dishes. When the cells reached $70 \%$ confluence, one of the dishes of cells was collected as the 0 day differentiation sample. At the same time, the medium was changed to differentiation medium (DM: DMEM + 2\% HS). Cells from one of the dishes was collected for RNA extraction, and the medium was changed in the other dishes on the 3rd, 6th, 9th, 12th, 15th, and 18th days.

\section{RNA extraction}

Total RNA was extracted from tissue and adhered cell samples using Trizol reagent (MRC, USA) or E.Z.N.Z. ${ }^{\mathrm{TM}}$ Total RNA Kit (Omega, United States). The RNA samples were digested by DNase I for 30 min to remove the genomic DNA before being reverse transcribed via PrimeScript ${ }^{\mathrm{TM}}$ RT Master Mix (Takara, Japan).

\section{Quantitative real-time polymerase chain reaction (PCR)}

Quantitative real-time PCR was carried out on the ABI 7500 RT-PCR system (Applied Biosystems, United States). Each $20-\mu \mathrm{L}$ reaction included $10 \mu \mathrm{L}$ SYBR ${ }^{\circledR}$ Premix Ex Taq ${ }^{\mathrm{TM}}$ II (Takara), $0.8 \mu \mathrm{L} 10 \mu \mathrm{M}$ PCR forward primer, $0.8 \mu \mathrm{L} 10 \mu \mathrm{M}$ PCR reverse primer, $0.4 \mu \mathrm{L}$ ROX 
Reference Dye (Takara), and $2 \mu \mathrm{L}$ template cDNA. The Bovine GAPDH (NM_001034034) gene was adopted as an endogenous control. Primers for the GAPDH gene and the genes involved in the Hh signaling pathway are listed in Table 1. The PCR protocol was as follows: initial denaturation step at $95^{\circ} \mathrm{C}$ for $30 \mathrm{~s}, 40$ cycles of denaturation at $95^{\circ} \mathrm{C}$ for $5 \mathrm{~s}$ and extension at $60^{\circ} \mathrm{C}$ for $34 \mathrm{~s}$ to amplify products, and a dissociation stage of $95^{\circ} \mathrm{C}$ for $15 \mathrm{~s}, 60^{\circ} \mathrm{C}$ for 1 min, and $95^{\circ} \mathrm{C}$ for $15 \mathrm{~s}$ to obtain the melting curve. All conditions were performed in triplicate based on a standard curve method. The $2^{-\Delta \Delta C t}$ method was applied to calculate fold changes of the expression levels of the genes between different tissue samples.

\begin{tabular}{ll} 
Table 1. Primers used for quantitative real-time polymerase chain reaction. \\
\hline Gene & Primer $\left(5^{\prime} \rightarrow 3^{\prime}\right)$ \\
\hline GAPDH & Forward primer: TTCAACGGCACAGTCAAGG \\
Gli 1 & Reverse primer: ACATACTCAGCACCAGCATCAC \\
Gli2 & Forward primer: TGCACATGCGCAGACACAC \\
& Reverse primer: TTACTGCAGCCCTCATGCTCAC \\
& Forward primer: GCAGCAGCAACTGTCTGAGTGA \\
\hline
\end{tabular}

\section{Statistical analysis}

Statistical analysis was performed using the SPSS 18.0 software. Statistical significance was determined using one-way analysis of variance, and $\mathrm{P}<0.05$ was considered to be statistically significant.

\section{RESULTS}

\section{Tissue-specific expression profile of Gli1 and Gli2}

Of the 13 examined tissues, Glil expression was the highest in abomasum (Figure 1A). Additionally, liver, lung, and small intestine also had high expression of Gli1 (Figure 1A). In contrast, the Gli2 expression level was high in abdominal fat and intestine (Figure 1B). No significant differences were observed between Gli1 and Gli2 expression in heart, kidney, rumen, reticulum, and omasum $(\mathrm{P}>0.05)$ (Figure 1). In the four stomachs, there was no significant difference in Gli2 expression ( $\mathrm{P}>0.05)$ (Figure 1B), but Gli1 transcript levels were much higher in the abomasum than in the other stomachs $(\mathrm{P}<0.05)$ (Figure 1A). Interestingly, the expression levels of Gli1 and Gli2 were the lowest in the muscle of the 13 examined tissues (Figure 1).

\section{Expression of Gli1 and Gli2 in different populations of muscle-derived cells}

Most of the cells in pp1 to pp6 were spindle shaped (Figure 2A-F), but the relative expression of Gli1 and Gli2 varied distinctively (Figure 2G-H). The relative expression of Gli1 showed a decreasing trend from pp1 to pp6 (Figure 2G). The Gli1 expression was lower in muscle tissue than in all muscle-derived cell populations $(\mathrm{P}<0.05)$ (Figure $2 \mathrm{G})$. Except pp2 and pp4 having higher Gli2 expression than pp1 $(\mathrm{P}<0.05)$ and pp3 $(\mathrm{P}<0.05)$, respectively, the expression of Gli2 decreased from pp1 to pp6, and the expression of Gli2 was lower in muscle than in other cell populations $(\mathrm{P}<0.05)$ (Figure $2 \mathrm{H})$. 

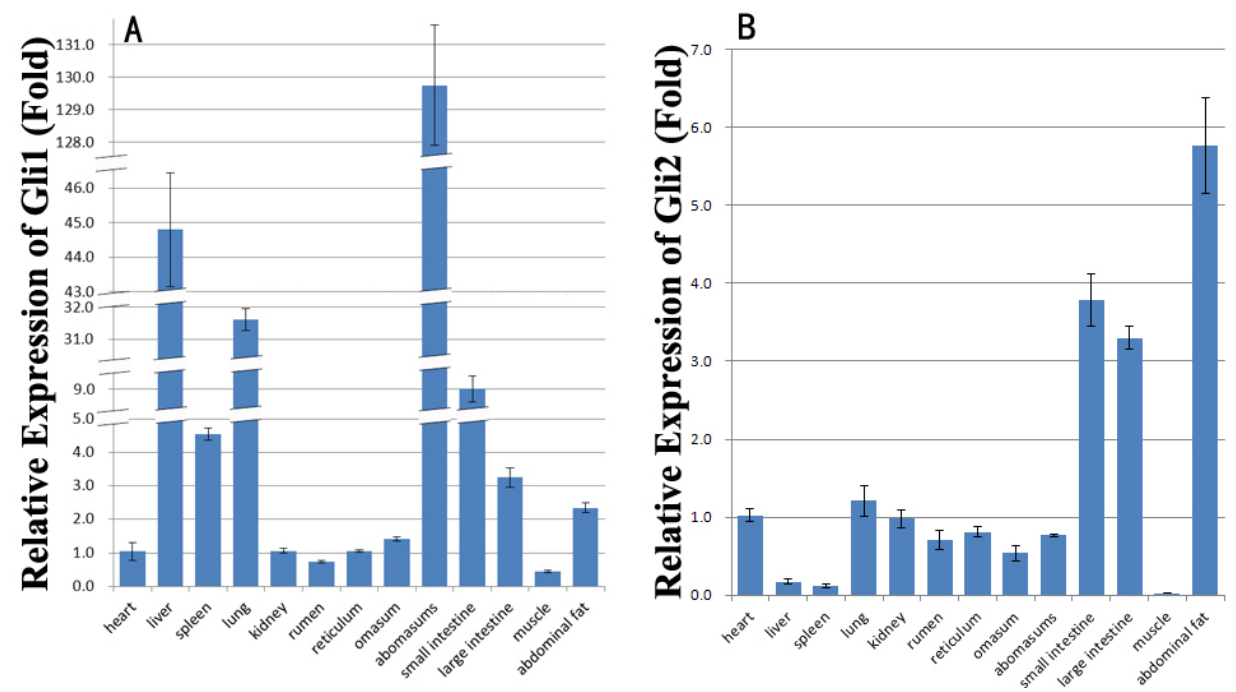

Figure 1. Tissue-specific expression profiles of Gli1 (A) and Gli2 (B).

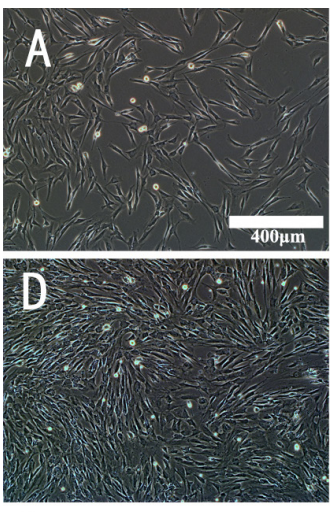

G
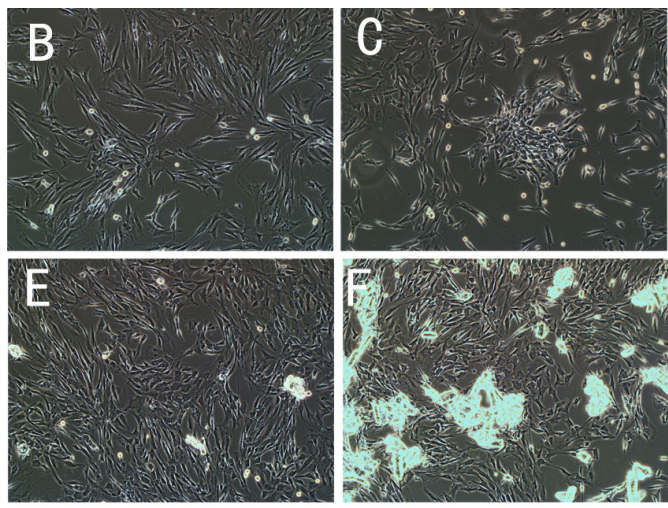

H
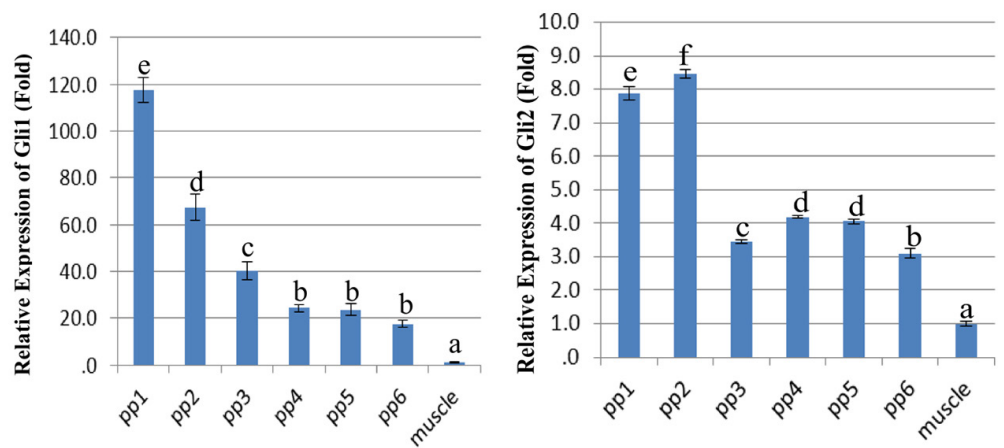

Figure 2. Different populations of muscle-derived cells and the relative expression levels of Gli1 and Gli2. A. preplate (pp)1; B. pp2; C. pp3; D. pp4; E. pp5; F. pp6; G. and H. Relative expression of Gli1 and Gli2 in different cell populations and muscle tissues. Different letters at the top of the columns indicate significant differences $(\mathrm{P}<0.05)$. 


\section{Expression of Gli1 and Gli2 during the myogenic differentiation of myoblasts}

Except on the 9th day of induced myogenic differentiation, the expression of Gli1 was lower in myoblasts than in muscle $(\mathrm{P}<0.05)$ (Figure $3 \mathrm{~A})$. During the process of myogenic differentiation, the expression of Glil fluctuated but was still elevated on the 3rd and 9th days and peaked on the 9th day (Figure 3A). Gli2 expression increased from the beginning to the 6th day of induced differentiation, and it declined from the 6th day to the 18th day except the 15th day (Figure 3B). The expression of Gli2 was much lower in muscle tissue than in all stages of myogenic differentiation of myoblasts $(\mathrm{P}<0.05)$, but the expression of Gli1 was relatively high in muscle tissue compared to its expression in myoblasts during the differentiation process (Figure 3).
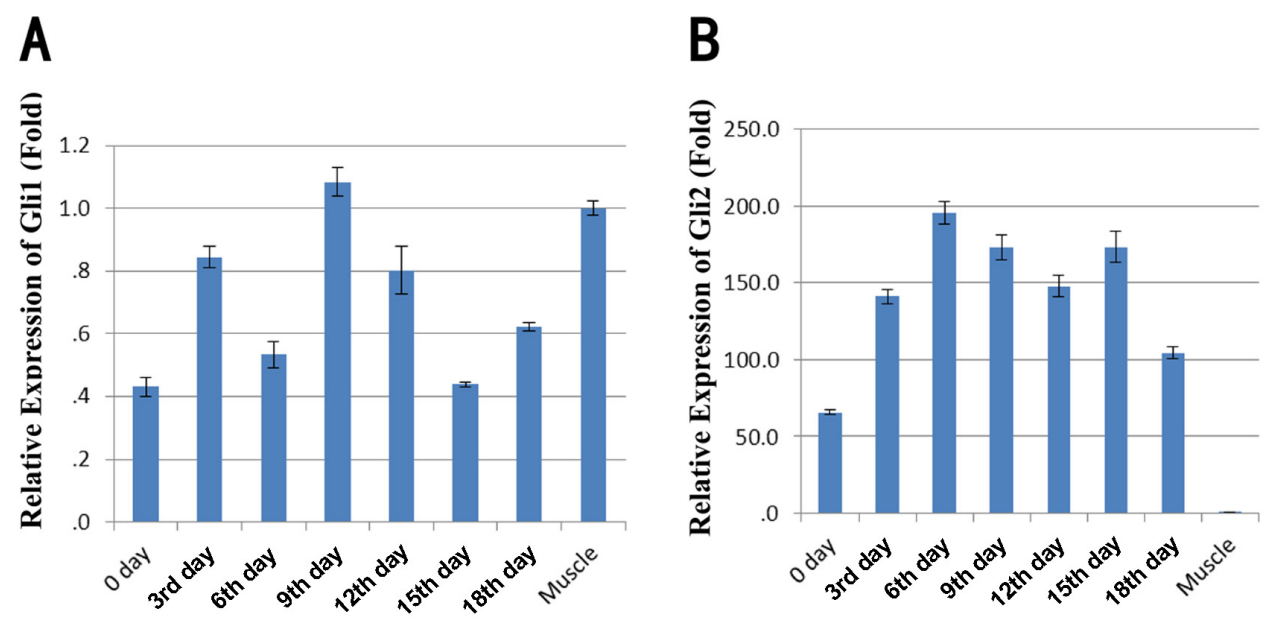

Figure 3. Expression of Gli1 and Gli2 in myoblasts during myogenic differentiation.

\section{DISCUSSION}

In vertebrates, there are three members of the Gli family, Gli1, Gli2, and Gli3, which are orthologs of the segment polarity gene Cubitus interruptus (Ci), a zinc-finger gene in Drosophila. Hh signaling modulates many kinds of cell activation by acting on Gli proteins to prevent the formation of repressor forms of Gli2 and Gli3 and to promote the activator forms of Gli1, Gli2, and Gli3 (Ribes and Briscoe, 2009). In Drosophila, Ci is a 155-kDa protein that contains five zinc fingers. The full-length $\mathrm{Ci}$ is the activator form, and the repressor form of $\mathrm{Ci}$ is a truncation of $75 \mathrm{kDa}$ (Cohen, 2003). The Gli family in vertebrates is less understood than $\mathrm{Ci}$ in Drosophila. Unlike $\mathrm{Ci}$, the repressor form of Gli1 does not undergo a proteolytic cleavage, and Gli1 does not appear to require binding with CBP for its activator form (Dai et al., 1999). It has been revealed that Gli1 does not have a repressor form, Gli2 has both activator and repressor forms, and Gli3 is a transcriptional repressor that balances and refines the activation of Gli1 and Gli2 (Ingham and McMahon, 2001; Nakajima et al., 2012).

The three Gli proteins in vertebrates make the Hh signaling more complex than in Drosophila, and Gli1 is not only the transcription factor but also the target gene of the Hh signaling pathway. Thus, cellular responses to Hh signaling might be modulated by the ex- 
pression of Gli genes, as well as the ligand concentration (Ingham and McMahon, 2001). The expression profiles of Gli1 and Gli2 in different tissues are quite distinct (Figure 1). Cohen (2003) reported that Glil has widespread expression in the mesoderm, especially in the ventral neural tube, and Gli2 also has widespread expression in the mesoderm, especially in the posterior neural tube, which overlaps with the expression of Gli3. Three Gli genes in vertebrates may have a complex relationship of competition and cooperation in different tissues and cells (Cohen, 2003). Although Hh signaling has been proven to influence almost all parts of the vertebrate body, in our research, the expression of Gli1 and Gli2, genes encoding two transcription factors of the Hh signaling pathway, are less identical in different tissues of cattle. Our results suggested that the response to Hh signaling was quite different in each tissue. We found that the expression levels of Glil and Gli2 were lower in muscle than all other endodermal organs. This corresponded with the general opinion that the Hh pathway is silent in postnatal animals (Straface et al., 2009).

Skeletal muscle is a structurally unique tissue consisting predominantly of highly specialized myofibers, which can generate force rapidly but lose the ability to proliferate. Therefore, a reserve pool of undifferentiated cells, which is normally quiescent but would be activated in response to turnover, growth demands, or damage of satellite cells, is important to the function of muscles. On the basis of the adhesion characteristics of different cell populations, a method of preplating cells onto collagen-coated plastic, which was mentioned previously, was established for primary cultures of muscle-derived stem cells (Qu et al., 1998). Fibroblasts and epithelial cells attach a short time after the heterogeneous cell suspension is plated on a Petri dish (Yaffe, 1968; Qu-Petersen et al., 2002). Almost 30 to 40\% of the cells in pp2 and pp3 are known to be nonmyogenic, whereas up to $95 \%$ of the cells in pp4 and pp5 express desmin (Qu et al., 1998). In our research, from pp1 to pp6, the expression of Gli1 and Gli2 decreased predominantly. Therefore, the decreased expression of Gli1 and Gli2, accompanied with the declining percentage of myoblasts, indicated that the Glil and Gli2 expression levels in myoblasts were lower than in fibroblasts or other cell populations derived from muscle.

Although the expression levels of Gli1 and Gli2 in muscle are both the lowest of the 13 tissues we investigated, many previous researches have testified that Hh signaling is crucial in muscle formation during embryonic and postnatal development of vertebrates (Flynt et al., 2007; Martin et al., 2007; Straface et al., 2009; Lobbardi et al., 2011; Hu et al., 2012). At the cellular level, the Hh signaling pathway promotes the proliferation and myogenic differentiation of myoblasts (Pola et al., 2003; Li et al., 2004; Koleva et al., 2005; Elia et al., 2007; Madhala-Levy et al., 2012). Several muscle regulatory factors, such as MyoD (Madhala-Levy et al., 2012), Myf5 (McDermott et al., 2005; Anderson et al., 2012), Pax7 (Madhala-Levy et al., 2012), and MyoG (Madhala-Levy et al., 2012), have been found to be regulated by Hh signaling. In bovine myoblasts, we observed elevated expression levels of Gli2 on the 3rd and 6th day of induced differentiation by serum starvation. The expression of Gli1 was elevated on the 3rd and 9th days, but its expression declined on the 6th day. A similar expression profile of Gli1 and Gli2 was observed in dimethyl sulfoxide-induced P19 cells (Voronova et al., 2013).

Gli1 and Gli2 are transcription factors of the Hh signaling pathway, and Gli1 is a target gene of this pathway as well. We speculated that Hh signaling was activated in neither myocytes nor quiescent myoblasts; instead, Hh signaling was activated in fibroblasts or other early attached cells of bovine muscle tissue. The elevated expression of Gli1 and Gli2 after serum starvation supports the reactivation of the Shh pathway after ischemia in the adult skeleton and myocardium (Pola et al., 2003; Kusano et al., 2005). Therefore, it suggested that $\mathrm{Hh}$ 
signaling would be activated while the quiescent bovine myoblast was stimulated to initiate myogenic differentiation.

\section{ACKNOWLEDGMENTS}

Research supported by the National Natural Science Foundation of China (\#31272411), the National Twelfth "Five Years" Science and Technology Support Projects (\#2011BAD28B04-03 and \#2011AA100307-02), the China National "863" Program (\#2013AA102505), the GMO New Varieties Major Project (\#2013ZX08007-002), and the National Beef and Yak Industrial Technology System (\#CARS-38).

\section{REFERENCES}

Anderson C, Williams VC, Moyon B, Daubas P, et al. (2012). Sonic hedgehog acts cell-autonomously on muscle precursor cells to generate limb muscle diversity. Genes Dev. 26: 2103-2117.

Bai CB, Stephen D and Joyner AL (2004). All mouse ventral spinal cord patterning by hedgehog is Gli dependent and involves an activator function of Gli3. Dev. Cell 6: 103-115.

Cai J and Deng L (2010). Regulations of Hedgehog signaling pathway on mesenchymal stem cells. Zhongguo Xiu. Fu Chong. Jian. Wai Ke Za Zhi 24: 993-996.

Cai J, Huang Y, Chen X, Xie H, et al. (2012a). Regulation of sonic hedgehog on vascular endothelial growth factor, basic fibroblast growth factor expression and secretion in bone marrow mesenchymal stem cells. Zhongguo Xiu. Fu Chong. Jian. Wai Ke Za Zhi 26: 112-116.

Cai JQ, Huang YZ, Chen XH, Xie HL, et al. (2012b). Sonic hedgehog enhances the proliferation and osteogenic differentiation of bone marrow-derived mesenchymal stem cells. Cell Biol. Int. 36: 349-355.

Cohen MM Jr (2003). The hedgehog signaling network. Am. J. Med. Genet. A 123A: 5-28.

Dai P, Akimaru H, Tanaka Y, Maekawa T, et al. (1999). Sonic Hedgehog-induced activation of the Gli1 promoter is mediated by GLI3. J. Biol. Chem. 274: 8143-8152.

Dwyer JR, Sever N, Carlson M, Nelson SF, et al. (2007). Oxysterols are novel activators of the hedgehog signaling pathway in pluripotent mesenchymal cells. J. Biol. Chem. 282: 8959-8968.

Elia D, Madhala D, Ardon E, Reshef R, et al. (2007). Sonic hedgehog promotes proliferation and differentiation of adult muscle cells: Involvement of MAPK/ERK and PI3K/Akt pathways. Biochim. Biophys. Acta 1773: 1438-1446.

Flynt AS, Li N, Thatcher EJ, Solnica-Krezel L, et al. (2007). Zebrafish miR-214 modulates Hedgehog signaling to specify muscle cell fate. Nat. Genet. 39: 259-263.

Fontaine C, Cousin W, Plaisant M, Dani C, et al. (2008). Hedgehog signaling alters adipocyte maturation of human mesenchymal stem cells. Stem Cells 26: 1037-1046.

Ho JE, Chung EH, Wall S, Schaffer DV, et al. (2007). Immobilized sonic hedgehog N-terminal signaling domain enhances differentiation of bone marrow-derived mesenchymal stem cells. J. Biomed. Mater. Res. A 83: 1200-1208.

Hooper JE and Scott MP (2005). Communicating with Hedgehogs. Nat. Rev. Mol. Cell Biol. 6: 306-317.

Hu JK, McGlinn E, Harfe BD, Kardon G, et al. (2012). Autonomous and nonautonomous roles of Hedgehog signaling in regulating limb muscle formation. Genes Dev. 26: 2088-2102.

Ingham PW and McMahon AP (2001). Hedgehog signaling in animal development: paradigms and principles. Genes Dev. 15: 3059-3087.

James AW, Leucht P, Levi B, Carre AL, et al. (2010). Sonic Hedgehog influences the balance of osteogenesis and adipogenesis in mouse adipose-derived stromal cells. Tissue Eng. Part. A 16: 2605-2616.

Koleva M, Kappler R, Vogler M, Herwig A, et al. (2005). Pleiotropic effects of sonic hedgehog on muscle satellite cells. Cell Mol. Life Sci. 62: 1863-1870.

Kusano KF, Pola R, Murayama T, Curry C, et al. (2005). Sonic hedgehog myocardial gene therapy: tissue repair through transient reconstitution of embryonic signaling. Nat. Med. 11: 1197-1204.

Li X, Blagden CS, Bildsoe H, Bonnin MA, et al. (2004). Hedgehog can drive terminal differentiation of amniote slow skeletal muscle. BMC Dev. Biol. 4: 9.

Li X, Wei G, Wang X, Liu DH, et al. (2012). Targeting of the Sonic Hedgehog pathway by atractylenolides promotes chondrogenic differentiation of mesenchymal stem cells. Biol. Pharm. Bull. 35: 1328-1335.

Lobbardi R, Lambert G, Zhao J, Geisler R, et al. (2011). Fine-tuning of Hh signaling by the RNA-binding protein Quaking 
to control muscle development. Development 138: 1783-1794.

Madhala-Levy D, Williams VC, Hughes SM, Reshef R, et al. (2012). Cooperation between Shh and IGF-I in promoting myogenic proliferation and differentiation via the MAPK/ERK and PI3K/Akt pathways requires Smo activity. J. Cell Physiol. 227: 1455-1464.

Martin BL, Peyrot SM and Harland RM (2007). Hedgehog signaling regulates the amount of hypaxial muscle development during Xenopus myogenesis. Dev. Biol. 304: 722-734.

McDermott A, Gustafsson M, Elsam T, Hui CC, et al. (2005). Gli2 and Gli3 have redundant and context-dependent function in skeletal muscle formation. Development 132: 345-357.

Milla LA, Cortés CR, Hodar C, Onate MG, et al. (2012). Yeast-based assay identifies novel Shh/Gli target genes in vertebrate development. BMC Genomics 13: 2.

Nakajima T, Iguchi T and Sato T (2012). Hedgehog signaling plays roles in epithelial cell proliferation in neonatal mouse uterus and vagina. Cell Tissue Res. 348: 239-247.

Oliveira FS, Bellesini LS, Defino HL, da Silva Herrero CF, et al. (2012). Hedgehog signaling and osteoblast gene expression are regulated by purmorphamine in human mesenchymal stem cells. J. Cell Biochem. 113: 204-208.

Persson M, Stamataki D, te Welscher P, Andersson E, et al. (2002). Dorsal-ventral patterning of the spinal cord requires Gli3 transcriptional repressor activity. Genes Dev. 16: 2865-2878.

Pola R, Ling LE, Aprahamian TR, Barban E, et al. (2003). Postnatal recapitulation of embryonic hedgehog pathway in response to skeletal muscle ischemia. Circulation 108: 479-485.

Qu-Petersen Z, Deasy B, Jankowski R, Ikezawa M, et al. (2002). Identification of a novel population of muscle stem cells in mice: potential for muscle regeneration. J. Cell Biol. 157: 851-864.

Qu Z, Balkir L, van Deutekom JC, Robbins PD, et al. (1998). Development of approaches to improve cell survival in myoblast transfer therapy. J. Cell Biol. 142: 1257-1267.

Ribes V and Briscoe J (2009). Establishing and interpreting graded Sonic Hedgehog signaling during vertebrate neural tube patterning: the role of negative feedback. Cold Spring Harb. Perspect. Biol. 1: a002014.

Schaefer GI, Perez JR, Duvall JR, Stanton BZ, et al. (2013). Discovery of small-molecule modulators of the Sonic Hedgehog pathway. J. Am. Chem. Soc. 135: 9675-9680.

Steinert AF, Weissenberger M, Kunz M, Gilbert F, et al. (2012). Indian hedgehog gene transfer is a chondrogenic inducer of human mesenchymal stem cells. Arthritis Res. Ther. 14: R168.

Straface G, Aprahamian T, Flex A, Gaetani E, et al. (2009). Sonic hedgehog regulates angiogenesis and myogenesis during post-natal skeletal muscle regeneration. J. Cell Mol. Med. 13: 2424-2435.

Varjosalo M and Taipale J (2008). Hedgehog: functions and mechanisms. Genes Dev. 22: 2454-2472.

Voronova A, Coyne E, Al MA, Fair JV, et al. (2013). Hedgehog signaling regulates MyoD expression and activity. J. Biol. Chem. 288: 4389-4404.

Warzecha J, Göttig S, Brüning C, Lindhorst E, et al. (2006). Sonic hedgehog protein promotes proliferation and chondrogenic differentiation of bone marrow-derived mesenchymal stem cells in vitro. J. Orthop. Sci. 11: 491-496.

Wu X, Cai ZD, Lou LM and Chen ZR (2013). The effects of inhibiting hedgehog signaling pathways by using specific antagonist cyclopamine on the chondrogenic differentiation of mesenchymal stem cells. Int. J. Mol. Sci. 14: 5966-5977.

Yaffe D (1968). Retention of differentiation potentialities during prolonged cultivation of myogenic cells. Proc. Natl. Acad. Sci. U. S. A. 61: 477-483. 